\title{
Hydroxychloroquine Metabolites - An Exploratory Computational Study
}

\author{
Mohammed Monirul Islam ${ }^{1, *}$, Purna Sivarama Singh ${ }^{2}$, Sayeed Rushd ${ }^{3}$ \\ ${ }^{1}$ Department of Biomedical Sciences, College of Clinical Pharmacy, King Faisal University,Al-Ahsa, SAUDI ARABIA. \\ 2Department of Biomedical Sciences, College of Medicine, King Faisal University, Al-Ahsa, SAUDI ARABIA. \\ ${ }^{3}$ Department of Chemical Engineering, College of Engineering, King Faisal University, AI-Ahsa, SAUDI ARABIA.
}

\begin{abstract}
Background: $\mathrm{HCQ}$ has been found to provide the immunomodulation that helps to abate the possible cytokine storm response caused by the SARS COVID-19 virus and subsequent failures of major organs. It is also being used historically in treating Malaria and Rheumatoid arthritis and is known to be useful in cancer treatment. However, it shows a half-life of up to 70 days and has severe adverse effects including gastric disorders and retinopathy. Hypothesis: Therefore, we propose to explore some of its metabolites/ intermediates namely Desethyl hydroxychloroquine (DHCQ), Desethyl chloroquine (DCQ), and Bis-desethyl chloroquine (BDCQ) as alternate drug systems with minimized toxicity. Results and Discussion: The structural and spectral studies of these lead compounds to be unraveled using computational modeling and the evaluation of ADME parameters such as absorption, distribution, metabolism, and excretion would be performed using the SwissADME tool to assess the drug-likeness and medicinal chemistry friendliness. The future scope of the work involves the docking studies of these compounds to assess the therapeutic compliance for the treatment of viral infections and rheumatoid arthritis.
\end{abstract}

Key words: Hydroxychloroquine metabolites, COVID-19 drugs, Computational, ADME studies, Rheumatoid arthritis, Immunomodulation.

\section{INTRODUCTION}

HCQ, a known antimalarial drug has assumed importance during the current pandemic COVID-19, due to its potential use in severe cases of infections. ${ }^{1,2}$ The current treatment protocol currently involves the management of symptoms and other antiviral support. HCQ, derived from CQ, has been found to provide immunomodulation which helps to abate the possible cytokine storm response caused by the virus and subsequent failures of major organs. ${ }^{1}$ Both CQ and HCQ reportedly hinder the binding of viruses onto cell membranes by altering the surface $\mathrm{pH}^{3}$ and further block the viral multiplication. Though CQ has been initially chosen as the antiviral drug for Covid, due to its availability and cost-effectiveness, an overdose of CQ can cause severe poisoning even leading to morbidity. ${ }^{4}$ On the other hand, HCQ, derived from CQ, has an equivalent drug efficacy but with a reduced toxicity impact. A recent comparative study on antiviral potency showed that HCQ provides a better antiviral effect than CQ in treating COVID-19. ${ }^{1,2}$ HCQ has also been explored recently in cancer treatment to inhibit auto phagocytosis or the self-devouring of body cells. ${ }^{5}$ Another major application of HCQ is for treating rheumatoid arthritis; however, it suffers from the major side effect of induced retinopathy ${ }^{6,7}$ and GI disorders. ${ }^{8}$

Both CQ and HCQ are 4-aminoquinoline derivatives with a planar aromatic core and a slightly basic side chain which helps in the intracellular lysosomal accumulation of drugs. ${ }^{9-11}$ These molecules possess chiral centers and hence are present as enantiomers. The $\mathrm{S}(+)$ enantiomer binds more strongly to the plasma protein as compared to the $\mathrm{R}(-)$ counterpart. ${ }^{12,13}$ Upon oral administration,
Submission Date: 10-10-2021; Revision Date: 14-11-2021; Accepted Date: 03-12-2021.

DOI: 10.5530/ijper.56.1.25 Correspondence: Dr. Mohammed Monirul Islam

Department of Biomedical Sciences, College of Clinical Pharmacy, King Faisal University, Al-Ahsa, 31982, SAUDI ARABIA

E-mail: mislam@kfu.edu.sa

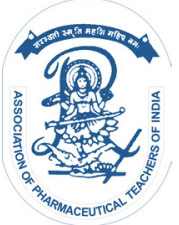

www.ijper.org 
HCQ undergoes CYP450 assisted metabolism to produce major metabolites namely DHCQ, DCQ, BDCQ. ${ }^{14}$ Among these DHCQ has been identified as the major metabolite even with a therapeutic value greater than HCQ itself while BDCQ has been found to cause the listed side effects. ${ }^{8}$

With the novel Coronavirus undergoing rapid genetic mutations, new treatment options and drug combinations are needed to be explored. Potential compounds with possible antiviral properties need to be identified and qualified to keep in mind the possibility of future pandemics and also to reduce the cost of treatment and hospitalizations. Computational studies often provide testable evaluations of probable drug systems.

In the present study the three metabolites of HCQ namely DHCQ, DCQ, BDCQ are selected for the in silico structural and spectral studies to understand the structure-activity relationships. These compounds are also subjected to Absorption, Distribution, Metabolism, and Excretion (ADME studies) computationally to assess the drug likeness and medicinal chemistry friendliness.

\section{MATERIALS AND METHODS DFT Calculations}

The ab-initio computational modeling for the optimization and spectral studies of the molecules were performed using the Gaussian09 software package. ${ }^{15}$ The graphical interface GaussView 6.0 was used for creating the input structures and then interpreting the output files.

\section{ADME Studies}

The ADME parameters were analyzed using the web tool SwissADME. ${ }^{16,17}$ The physiochemical properties of the molecules were evaluated by using the bio-availability radar and the BOILED- Egg plots available with the program.

\section{RESULTSDFT STUDIES}

\section{Structural Optimization}

The optimized structures were computed with Gaussian 09 using the Density Functional Theory approach and B3LYP/6-311 basis sets. The optimized structures of DHCQ, DCQ and BDCQ corresponding to the global minimum energies were appended below with atom labeling (Figure 1). The global minimum energies were computed at $-1322.58,-1247.3$ and -1168.75 a.u for DHCQ, DCQ, and BDCQ respectively while the corresponding dipole moments were calculated at 6.66, 6.41 and 6.55 D. Similarly, the polarizabilities of the metabolites were computed at 206.9, 204.23, and 188.39

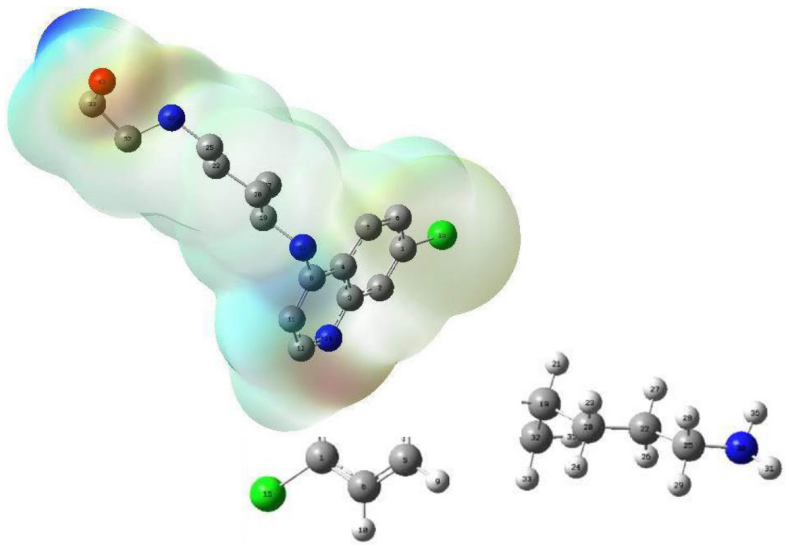

Figure 1: Optimized geometrical structures of DHCQ (1), DCQ (2), and BDCQ (3) at DFTB3LYP/6-311g basis set.

Bohr ${ }^{3}$ for DHCQ, DCQ, and BDCQ respectively. Both dipole moments and polarizabilities are useful indicators for molecular docking investigations. ${ }^{3}$

The three-dimensional molecular geometry and the corresponding geometrical parameters derived computationally reveal useful information regarding the drug-receptor interactions. The geometrical parameters such as bond lengths and bond angles of the metabolites are computed and presented below in Table 1. The computed parameters are then juxtaposed against the experimentally derived data corresponding to the parent molecule $\mathrm{CQ}^{18}$ for comparison.

\section{Frontier Orbital Analysis}

The frontier molecular orbitals namely Highest Occupied Molecular Orbitals (HOMO) and Lowest Occupied Molecular Orbitals (LUMO) determine the extent of interaction of drug systems with receptors and other molecules. ${ }^{3}$ HOMO and LUMO energies describe the electron releasing and electron-withdrawing capabilities respectively and depict the thermodynamical stability and chemical reactivity of the systems. ${ }^{19}$ These are useful descriptors in gauging the propensity of $\mathrm{HOMO}$ to LUMO electron transfer and hence the stability of the systems. The detailed HOM- LUMO profiles of DHCQ, DCQ, and BDCQ and global reactivity descriptors such as electronic chemical potential, absolute electro negativity, global softness, global hardness, and electrophilicity are presented in Table 2. According to Koopman's theorem, the ionization potential (I), and electron affinity (A) are calculated as the negative values of the energy of the highest occupied molecular orbital and the lowest unoccupied molecular orbital respectively. 


\begin{tabular}{|c|c|c|c|c|}
\hline Bonds & DHCQ & DCQ & BDCQ & Exptl. \\
\hline C1-C2 & 1.369 & 1.369 & 1.369 & 1.374 \\
\hline C1-C6 & 1.411 & 1.411 & 1.411 & 1.411 \\
\hline C1-Cl15 & 1.831 & 1.831 & 1.831 & 1.755 \\
\hline $\mathrm{C} 2-\mathrm{C} 3$ & 1.420 & 1.420 & 1.420 & 1.500 \\
\hline C3-C4 & 1.433 & 1.433 & 1.433 & 1.432 \\
\hline C3-N14 & 1.377 & 1.377 & 1.377 & 1.344 \\
\hline C4-C5 & 1.420 & 1.420 & 1.420 & 1.418 \\
\hline $\mathrm{C} 4-\mathrm{C} 8$ & 1.431 & 1.431 & 1.431 & 1.442 \\
\hline C5-C6 & 1.378 & 1.378 & 1.378 & 1.374 \\
\hline C8-C11 & 1.385 & 1.384 & 1.385 & 1.393 \\
\hline C8-N17 & 1.433 & 1.434 & 1.434 & 1.371 \\
\hline C11-C12 & 1.414 & 1.414 & 1.414 & 1.407 \\
\hline C12-N14 & 1.329 & 1.329 & 1.329 & 1.368 \\
\hline N17-C19 & 1.501 & 1.501 & 1.501 & 1.500 \\
\hline C19-C20 & 1.540 & 1.540 & 1.540 & - \\
\hline C19-C38 & 1.530 & 1.530 & 1.530 & 1.546 \\
\hline C20-C22 & 1.537 & 1.537 & 1.536 & 1.534 \\
\hline C22-C25 & 1.538 & 1.538 & 1.535 & 1.554 \\
\hline C25-N30 & 1.467 & 1.469 & 1.467 & 1.469 \\
\hline N30-C32 & 1.465 & 1.470 & - & 1.498 \\
\hline C32-C33 & 1.516 & 1.529 & - & 1.319 \\
\hline C33-O42 & 1.464 & - & - & - \\
\hline C2-C1-C6 & 122.624 & 122.624 & 122.613 & 121.720 \\
\hline C2-C1-Cl15 & 119.385 & 119.377 & 119.375 & 119.400 \\
\hline C6-C1-Cl15 & 117.986 & 117.993 & 118.005 & 118.800 \\
\hline $\mathrm{C} 1-\mathrm{C} 2-\mathrm{C} 3$ & 118.982 & 118.980 & 118.990 & 120.350 \\
\hline C2-C3-C4 & 119.648 & 119.649 & 119.655 & 119.100 \\
\hline C2-C3-N14 & 117.706 & 117.719 & 117.692 & 116.900 \\
\hline C4-C3-N14 & 122.639 & 122.625 & 122.645 & 123.200 \\
\hline C3-C4-C5 & 118.741 & 118.753 & 118.730 & 118.200 \\
\hline C3-C4-C8 & 117.836 & 117.838 & 117.822 & 117.700 \\
\hline C5-C4-C8 & 123.386 & 123.374 & 123.410 & 124.000 \\
\hline C4-C5-C6 & 120.895 & 120.887 & 120.900 & 121.700 \\
\hline C1-C6-C5 & 119.041 & 119.044 & 119.048 & 119.000 \\
\hline C4-C8-C11 & 118.029 & 118.052 & 118.040 & 116.800 \\
\hline C4-C8-N17 & 120.203 & 120.108 & 120.212 & 120.800 \\
\hline C11-C8-N17 & 121.744 & 121.817 & 121.722 & 124.300 \\
\hline C8-C11-C12 & 120.140 & 120.123 & 120.143 & 119.700 \\
\hline C11-C12-N14 & 123.603 & 123.601 & 123.595 & 125.200 \\
\hline C3-N14-C12 & 117.673 & 117.686 & 117.679 & 116.000 \\
\hline C8-N17-C19 & 118.713 & 118.689 & 118.763 & 124.700 \\
\hline N17-C19-C20 & 107.266 & 107.266 & 107.195 & 113.500 \\
\hline N17-C19-C38 & 111.850 & 111.799 & 111.894 & 108.900 \\
\hline C20-C19-C38 & 112.686 & 112.716 & 112.731 & 113.800 \\
\hline C19-C20-C22 & 114.598 & 114.598 & 114.607 & 115.900 \\
\hline C20-C22-C25 & 112.312 & 112.272 & 112.730 & 112.500 \\
\hline C22-C25-N30 & 112.597 & 112.514 & 110.789 & 113.400 \\
\hline C25-N30-C32 & 116.557 & 116.675 & 114.293 & 112.200 \\
\hline C30-C32-C33 & 109.822 & 110.840 & 110.797 & 113.300 \\
\hline C32-C33-O42 & 106.395 & - & - & - \\
\hline
\end{tabular}

\begin{tabular}{|c|c|c|c|}
\hline \multicolumn{4}{|c|}{ Table 2: Global reactivity parameters. } \\
\hline Parameters & DHCQ & DCQ & BDCQ \\
\hline ELOMO & -1.849 & -1864 & -1.866 \\
\hline EHOMO & -5.869 & -5.945 & -6.219 \\
\hline ELUMO - EHOMO & 4.019 & 4.081 & 4.353 \\
\hline Ionization potential $(\mathrm{I})$ & 1.849 & 1.864 & 1.866 \\
\hline Electron affinity $(\mathrm{A})$ & 5.869 & 5.945 & 6.219 \\
\hline Global hardness $(\eta)$ & 2.01 & 2.04 & 2.17 \\
\hline Global softness $(\mathrm{S})$ & 0.248 & 0.245 & 0.229 \\
\hline Electrophilicity $(\omega)$ & 3.704 & 3.734 & 3.753 \\
\hline Chemical potential $(\mu)$ & -3.859 & -3.904 & -4.042 \\
\hline $\begin{array}{c}\text { Absolute electro } \\
\text { negativity }(\mathrm{X})\end{array}$ & 3.859 & 3.904 & 4.042 \\
\hline $\begin{array}{c}\text { Maximum charge transfer } \\
\text { index }(\Delta \text { Nmax })\end{array}$ & 1.919 & 1.913 & 1.862 \\
\hline
\end{tabular}

The absolute electronegativity $(\chi)$ is calculated using the equation $\chi=\frac{\mathrm{I}+\mathrm{A}}{2}$ and the electronic chemical potential $(\mu)$ is computed as $\mu=-\chi$. The value of global hardness $(\eta)$ and softness (S) were obtained using the equations $\eta=\frac{\text { ELUMO }- \text { EHOMO }}{2}$ and $S=\frac{1}{2 \eta}$ respectively. The electrophilicity $(\omega)$ of the compounds were calculated using $\omega=\frac{\mu}{2 \eta}$ while another important parameter the maximum charge transfer index was calculated by the formula $\Delta \mathrm{Nmax}=\frac{-\mu}{\eta}$

As the higher value of HOMO energy and the lower value of LUMO energy imply better electron donating and accepting tendencies respectively, it is evident that DHCQ has the most electron- donating tendency while BDCQ has the most electron-withdrawing tendency. The HOMO-LUMOenergy gap of all the three molecules is more or less comparable and DHCQ shows the least valuewhile BDCQ showing a relatively larger band gap. Further BDCQ demonstrates the highest ionization potential and electron affinity indicating the least chemical reactivity. Conversely, DHCQ has the lowest ionization potential and electron affinity making it the most reactive system. This was further corroborated by the lowest value of the HOMO-LUMO energy gap for DHCQ. Electrophilicity index ( $\omega)$, which reflects the reduction of energy during the maximal flow of electrons between two systems ${ }^{20}$ would show us whether the species is capable of chargedonation. ${ }^{21}$ As a thumb rule, a species with lesser ' $\omega$ ' would be more reactive and 
nucleophilic and those with a higher value would go on to become a better electrophilic system. The studies revealed that DHCQ promises to be a better nucleophile while BDCQ showed a better propensity of an electrophile. This implies that BDCQ will be able to receive an electron doublet so as to react with other nucleophilic systems. Both DHCQ and DCQ demonstrated lower values of absolute electro negativity $(\chi)$ making them more basic as compared to BDCQ. This is an important aspect considering the fact that the basicity of the side chain governs the intracellular lysosomal activity of the drug molecules.

The three-dimensional HOMO- LUMO plots of the molecules are illustrated in Figure 2. It is evident that the HOMO density is populated over the aromatic core and slightly onto the chlorine and nitrogen of the side chain with no population seen over the rest of the basic side chain. On the other hand, the LUMO population is by large populated over the whole basic side chain with no apparent population over the aromatic core.

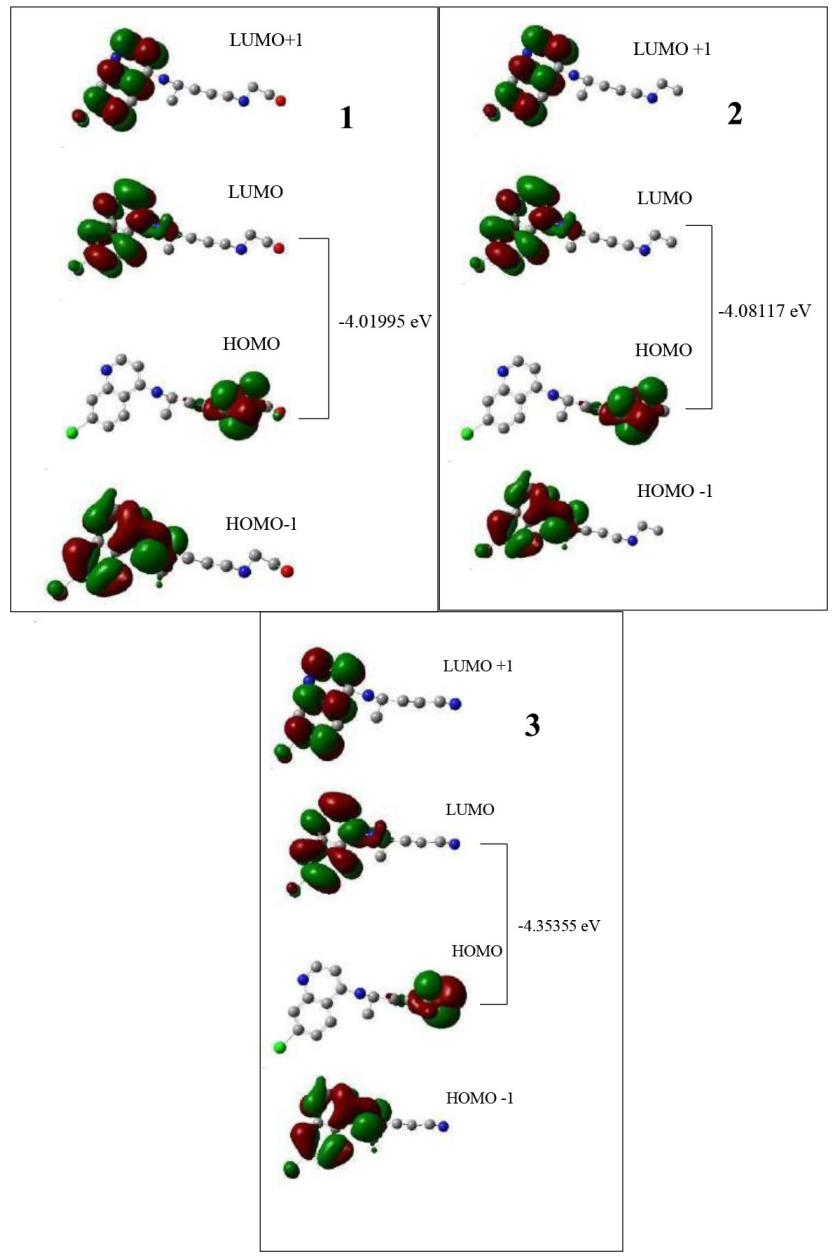

Figure 2: HOMO-LUMO Energy gap of DHCQ (1), DCQ (2), and BDCQ (3).
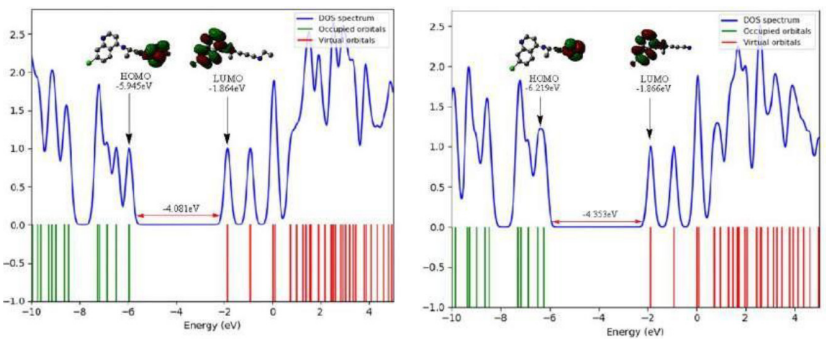

Figure 3: DOS Plot for DHCQ, DCQ and BDCQ (Clockwise order).

In the HOMO-LUMO diagram, the green and red colors symbolize the negative and positive charge fields respectively. This can be further substantiated by plotting the Density of States (DOS) plot which describes the number of states available at various energy levels (Figure 3). The DOS plots also visualize the occupied and virtual orbitals of the molecules and also compute the HOMO-LUMO energy gap. The relatively lower values of HOMO-LUMO energies of DHCQ, DCQ, and BDCQ at 4.019, 4.081, and $4.353 \mathrm{eV}$ respectively indicate ease of transfer of electrons in the molecules.

\section{Mulliken Charge Analysis}

The Mulliken atomic charges demonstrate the electron charge distributions in molecules. The mostnegative site of the molecule will be the one most susceptible for an electrophilic attack while themost positive sites will be more liable for nucleophilic attack. The Mulliken analysis for the title molecules was performed computationally at DFT-B3LYP/6.311g and the results were tabulated in Table 3.

Our results showed N17 and O42 positions as the most negative sites for DHCQ and N17 and N30 positions for both DCQ and BDCQ indicating possibilities of electrophilic attacks at these sites. Conversely, the C8 position has been found to the most positive and hence liable for nucleophilic attack in all three molecules. The structure-activity relationship of potential drug molecules including susceptibility towards nucleophilic and electrophilic attack and other intermolecular interactions can also be validated by performing molecular electrostatic potential (MEP) studies.

MEP plots help to picturize regions of variable charges by using color grading. It also envisages the size, shape, and reactive behaviors of molecules. These threedimensional diagrams visualize negative, positive, and neutral electrostatic potential regions by virtue of the color gradient; with red and blue colors representing the most electronegative and electropositive regions respectively. The regions with zero potential are shown in green color. The MEP plots of DHCQ, DCQ 


\begin{tabular}{|c|c|c|c|}
\hline Atom label & DHCQ & DCQ & BDCQ \\
\hline $1 \mathrm{C}$ & -0.284 & -0.284 & -0.284 \\
\hline $2 C$ & -0.007 & -0.007 & -0.007 \\
\hline $3 C$ & -0.002 & -0.002 & -0.003 \\
\hline $4 C$ & 0.014 & 0.015 & 0.015 \\
\hline $5 C$ & -0.058 & -0.057 & -0.058 \\
\hline $6 C$ & -0.080 & -0.080 & -0.080 \\
\hline $8 C$ & 0.244 & 0.241 & 0.244 \\
\hline $11 \mathrm{C}$ & -0.192 & -0.191 & -0.193 \\
\hline $12 \mathrm{C}$ & 0.022 & 0.022 & 0.022 \\
\hline $14 N$ & -0.354 & -0.354 & -0.354 \\
\hline $15 \mathrm{Cl}$ & -0.021 & -0.022 & -0.022 \\
\hline $17 N$ & -0.669 & -0.669 & -0.669 \\
\hline $19 \mathrm{C}$ & -0.044 & -0.043 & -0.044 \\
\hline $20 \mathrm{C}$ & -0.348 & -0.347 & -0.346 \\
\hline $22 \mathrm{C}$ & -0.324 & -0.319 & -0.334 \\
\hline $25 C$ & -0.192 & -0.196 & -0.175 \\
\hline $30 N$ & -0.576 & -0.560 & -0.715 \\
\hline $32 \mathrm{C}$ & -0.171 & -0.181 & \\
\hline $33 C$ & -0.105 & -0.531 & \\
\hline $37 C$ & -0.500 & -0.501 & \\
\hline 420 & -0.619 & & \\
\hline
\end{tabular}

and BDCQ were generated computationally and are presented below in Figure 4.

\section{Spectral Studies}

Even though spectral studies are not always considered for geometrical and structural optimization, FTIR data can corroborate the optimized geometry by providing positive force field constants. Here, the FTIR scans of the title molecules were performed computationally using the DFT- B3LYP/6-311g basis set. The computed FTIR spectra of the molecules showed the characteristic peaks as expected (Figure 5). There were no negative peaks reported suggesting that the most optimized structures predicted were indeed corresponding to the global minimum energy. DHCQ showed the characteristic $\mathrm{OH}$ stretching vibration at $3700 \mathrm{~cm}^{-1}$ and a broad peak at around $1600 \mathrm{~cm}^{-1}$ corresponding to $\mathrm{C}=\mathrm{C}$ and $\mathrm{C}=\mathrm{N}$ stretching modes. The notable $\mathrm{C}-\mathrm{Cl}$ peak has been credited to the high intense peak around $650 \mathrm{~cm}^{-1}$. DCQ showed the typical $\mathrm{CH}$ vibrations around the $3000 \mathrm{~cm}^{-1}$ mark and $\mathrm{C}-\mathrm{Cl}$ peak close to $600 \mathrm{~cm}^{-1}$. The characteristic twin peaks for the terminal amino group have been visualized at $3500 \mathrm{~cm}^{-1}$, followed by $\mathrm{CH}$ stretching modes at around $3000 \mathrm{~cm}^{-1}$. Other notable

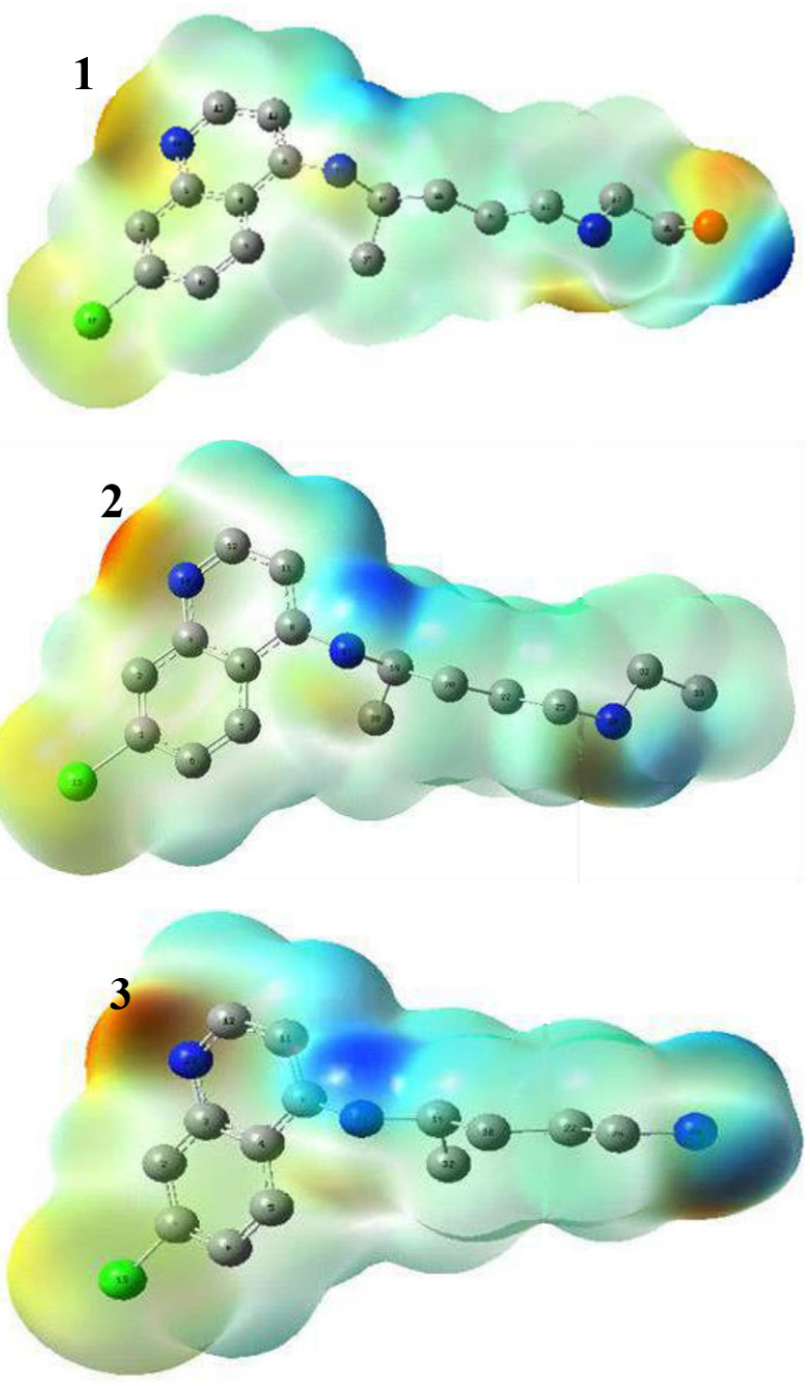

Figure 4: MEP plots of DHCQ (1), DCQ 92) and BDCQ (3).
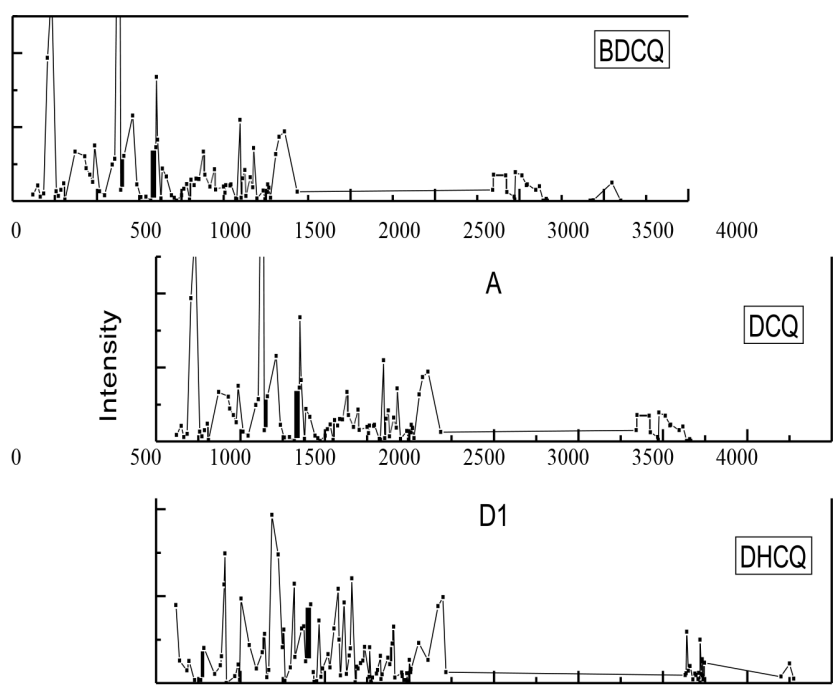

$\begin{array}{lllllllll}0 & 500 & 1000 & 1500 & 2000 & 2500 & 3000 & 3500 & 4000\end{array}$

Wavelength $\left(\mathrm{Cm}^{-1}\right)$

Figure 5: The consolidated FTIR spectra of DHCQ, DCQ and BDCQ computed at DFT-B3LYP/6-311g. 
peaks were noted at 1650 and $700 \mathrm{~cm}^{-1}$ corresponding to $\mathrm{C}-\mathrm{N}$ and $\mathrm{C}-\mathrm{Cl}$ stretching modes.

\section{ADME Studies}

The evaluation of ADME parameters namely Absorption, Distribution, Metabolism and Excretion provide crucial pre-clinical information to assess the suitability drug-like molecules. It is imperative that the potential drug system reaches the target site in the stipulated amount and be available in the correct quantity till its pharmacological functions are performed. ${ }^{22}$ The ADME parameters of DHCQ, DCQ, and BDCQ were assessed using the SwissADME web tool to evaluate the flexibility, lipophilicity, size, polarity, solubility, and saturation. The critical limits of the above-listed parameters were visualized using the bioavailability radar (Table 4). It also provides useful acumen regarding pharmacological attributes namely gastrointestinal absorption (HIA), blood-brain-barrier (BBB) permeability, topological polar surface area (TPSA), etc. The bioavailability radars of DHCQ, DCQ, BDCQ and the parent molecules such as CQ and HCQ were depicted in Figure 6 for comparison.

From Table 4, it is evident that none of the lead molecules or parent drugs showed any Lipinsky violations indicating overall druglikeness. However, DHCQ and DCQ showed one potential violation in the leadlikeness index while BDCQ showed none. The overall physiochemical parameters of the metabolites and drug systems are comparable. Of the three metabolites, DHCQ possesses 3 hydrogen bond acceptors and 3 hydrogen bond receptors making it more reactive with the receptor sites. Conversely DCQ and BDCQ possess 2 each of these. The results reveal that both DHCQ and BDCQ have lower logP values compared to DCQ and the parent drugs. This is advantageous as the toxicity of the drug systems decreases as $\log \mathrm{P}$ value decreases and systems with $\log \mathrm{P}$ value around 2.2 are more suitable for oral administration. ${ }^{23-25}$ It is worth noting that CQ has a $\log \mathrm{P}$ value around 4.2 well above the toxicity cut-

\begin{tabular}{|c|c|c|c|c|c|}
\hline Molecule & $\mathbf{C Q}$ & HCQ & DHCQ & DCQ & BDCQ \\
\hline MW & 319.87 & 335.87 & 307.82 & 291.82 & 263.77 \\
\hline \#Heavy atoms & 22 & 23 & 21 & 20 & 18 \\
\hline \#Aromatic heavy atoms & 10 & 10 & 10 & 10 & 10 \\
\hline \#Rotatable bonds & 8 & 9 & 8 & 7 & 5 \\
\hline \#H-bond acceptors & 2 & 3 & 3 & 2 & 2 \\
\hline \#H-bond donors & 1 & 2 & 3 & 2 & 2 \\
\hline Molar refractivity, MR & 97.41 & 98.57 & 88.86 & 87.7 & 77.99 \\
\hline TPSA & 28.16 & 48.39 & 57.18 & 36.95 & 50.94 \\
\hline Consensus Log P & 4.14 & 3.29 & 2.77 & 3.58 & 2.83 \\
\hline ESOL Log S & -4.55 & -3.91 & -3.31 & -3.95 & -3.4 \\
\hline Solubility & Moderate & Soluble & Soluble & Soluble & Soluble \\
\hline Gl absorption & High & High & High & High & High \\
\hline BBB permeant & Yes & Yes & Yes & Yes & Yes \\
\hline Pgp substrate & No & No & Yes & No & No \\
\hline CYP1A2 inhibitor & Yes & Yes & Yes & Yes & Yes \\
\hline CYP2C19 inhibitor & No & No & No & Yes & Yes \\
\hline CYP2C9 inhibitor & No & No & No & No & No \\
\hline CYP2D6 inhibitor & Yes & Yes & Yes & Yes & Yes \\
\hline CYP3A4 inhibitor & Yes & Yes & Yes & Yes & Yes \\
\hline $\log \mathrm{Kp}(\mathrm{cm} / \mathrm{s})$ & -4.96 & -5.81 & -6.23 & -5.38 & -5.84 \\
\hline Lipinski \#violations & 0 & 0 & 0 & 0 & 0 \\
\hline Bioavailability Score & 0.55 & 0.55 & 0.55 & 0.55 & 0.55 \\
\hline PAINS \#alerts & 0 & 0 & 0 & 0 & 0 \\
\hline Brenk \#alerts & 0 & 0 & 0 & 0 & 0 \\
\hline Leadlikeness \#violations & 2 & 2 & 1 & 1 & 0 \\
\hline Synthetic Accessibility & 2.76 & 2.82 & 2.6 & 2.54 & 2.35 \\
\hline
\end{tabular}



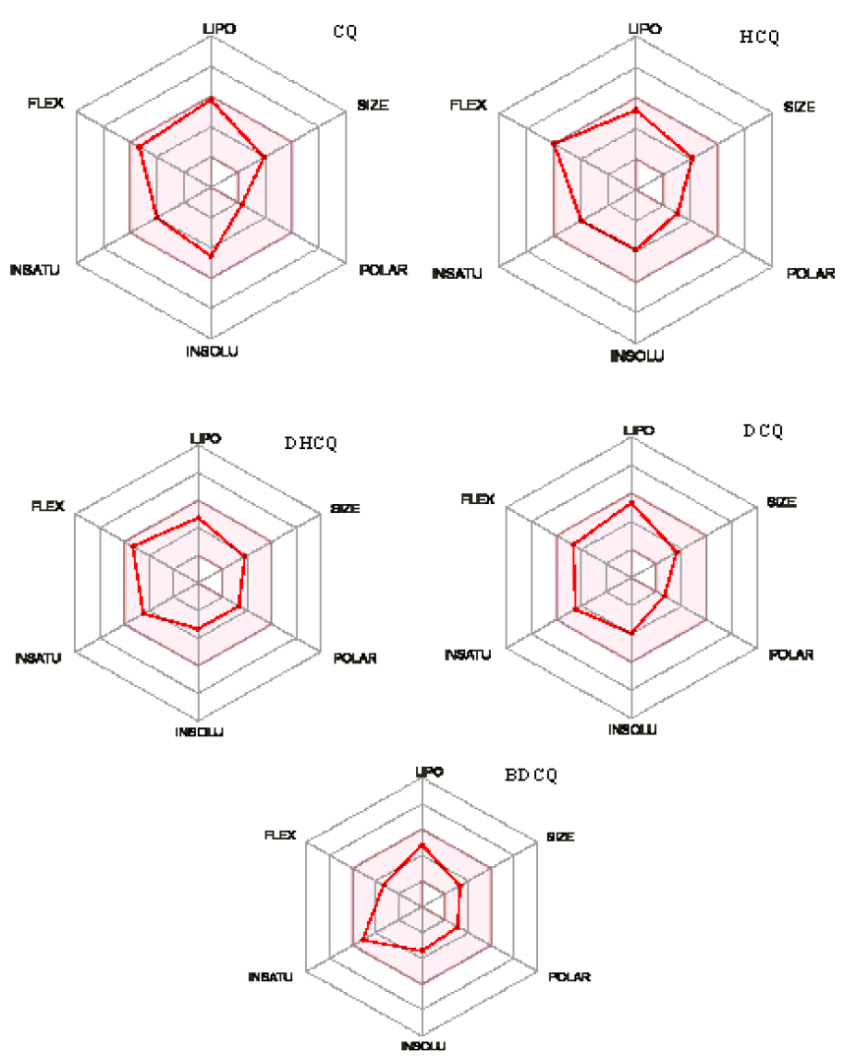

Figure 6: Bio-availability radars of the metabolites and parent drugs.

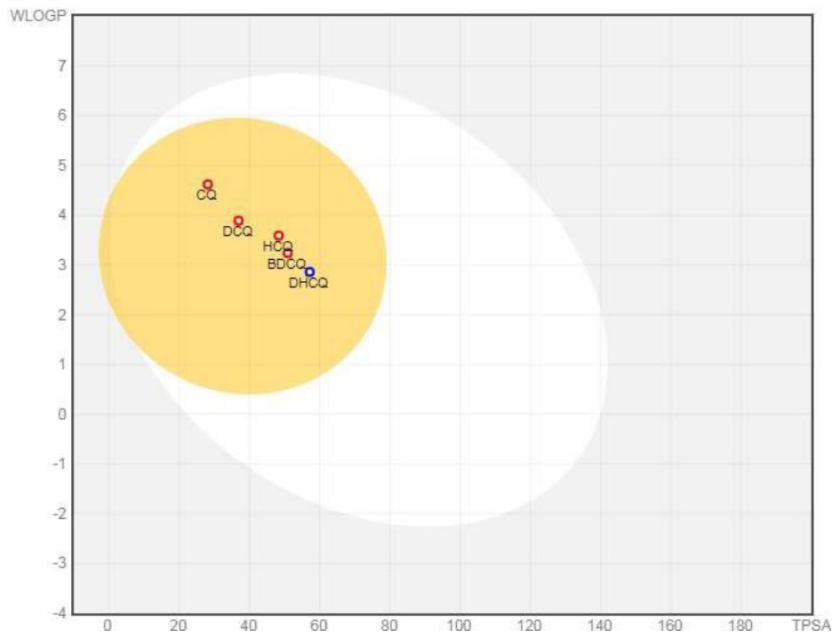

Figure 7: BOILED-Egg plot for the metabolites and parent drug molecules.

off limit of 3.0. Among the metabolites and parent molecules, DHCQ possesses the highest TPSA value due to the presence of polar hydroxyl group in the terminal position of the side chain. Studies revealed that an increased TPSA value and a lower $\log \mathrm{P}$ value ensure better bioavailability of the drug. The value of TPSA is an important parameter considering the fact that as
TPSA increases the blood-brain-barrier (BBA) index decreases. However, all the five molecules considered here have TPSA values well below the cut-off value of $90[\AA]^{2}$ making them BBB permeant. The metabolites, by and large, showed comparable inhibitory actions towards the major cytochromes as compared to the parent drugs.

The BOILED-Egg plot is the graphical output of the SwissADME software which showcases the gastrointestinal absorption and CNS absorption rates of the potential drug molecules Figure 7. It is inferred that those systems that appear in the egg's white would exhibit better gastro-intestinal absorption and those in the yellow yolk are better drugs for the central nervous system applications. In the BOILED-Egg plot, the molecules located in the 'egg white' will have more GI absorption, whereas those in the 'yolk' have more BBB permeation and CNS penetration. Molecules in the grey area have often shown poor activity as GI or CNS drugs. Further, the compounds that are colored blue get pumped out of the brain while those in red remain in the brain. The BOILED-Egg plot suggests that both the metabolites and the parent drugs have BBB permeation and have an impact on the CNS system.

\section{CONCLUSION}

Though both CQ and HCQ are known to possess good antiviral and anti-arthritis properties, they suffer from various side effects and prolonged half-life. Three major metabolites of HCQ namely DHCQ, DCQ, and BDCQ were selected for structural, spectral, and physiochemical assessment computationally. The optimized structure and geometrical parameters were computed, and the data showed concordance with the experimental results available in the literature. The HOM-LUMO energies of DHCQ, DCQ and BDCQ were computed along with the global reactivity descriptors such as electronic chemical potential, absolute electro negativity, global softness, global hardness, electrophilicity. Of the three metabolites, DHCQ showed the lowest ionization potential and electron affinity making it the most reactive system. This was further substantiated by having a lower HOMO-LUMO energy gap. DHCQ also exhibited the lowest value of absolute electro negativity $(\chi)$ making it more basic. This is an important aspect considering that the basicity of the side chain governs the intracellular lysosomal activity of the drug molecules. The Mulliken charge analysis showed N17 and O42 positions as the most negative sites for DHCQ and N17 and 
N30 positions for both DCQ and BDCQ indicating possibilities of electrophilic attacks at these sites.

The assessment of ADME properties revealed that none of the metabolites showed any Lipinsky violations indicating good overall drug likeness. Of the three metabolites, DHCQ possesses 3 hydrogen bond acceptors and 3 hydrogen bond receptors making it more reactive with the receptor sites. The results reveal that both DHCQ and BDCQ have lower log P values compared to the parent drugs which make them less toxic and suitable for oral intake. Both metabolites and parent drugs demonstrated very low values of TPSA and hence increased BBB permeation. In general, it can be concluded that DHCQ may be considered as a better lead molecule for the potential drug development and docking studies compared to DCQ and BDCQ.

\section{Funding}

This research was funded by the Deanship of Scientific Research at King Faisal University, Al- Ahsa, Saudi Arabia (Nasher Track Grant No. 206027).

\section{ACKNOWLEDGEMENT}

The authors acknowledge the Deanship of Scientific Research at King Faisal University, Kingdom of Saudi Arabia for their financial support under Nasher Track (Grant No. 206027) and their encouragement.

\section{CONFLICT OF INTEREST}

The authors declare no conflict of interest.

\section{ABBREVIATIONS}

HCQ: Hydroxychloroquine; CQ: Chloroquine; SARS: Severe acute respiratory syndrome; DHCQ: Desethyl hydroxychloroquine; DCQ: Desethyl chloroquine; BDCQ: Bis-desethyl chloroquine; ADME: absorption, distribution, metabolism, and excretion; TPSA: Topological polar surface area; BBB: Blood-brainbarrier; HOMO: Highest occupied molecular orbital; LUMO: Lowest unoccupied molecular orbital; GI: Gastrointestinal; DFT: Density functional theory; MEP: Molecular electrostatic potential; HIA: Gastrointestinal absorption; FTIR: Fourier-transform infrared spectroscopy.

\section{REFERENCES}

1. Yao X, Ye F, Zhang M, Cui C, Huang B, Niu P, et al. In vitro antiviral activity and projection of optimized dosing design of hydroxychloroquine for the treatment of severe acute respiratory syndrome coronavirus 2 (SARS-
CoV-2). Clin Infect Dis. 2020;71(15):732-9. doi: 10.1093/cid/ciaa237, PMID 32150618.

2. Liu K, Tang M, Liu Q, Han X, Jin H, Zhu H, et al. Bi-directional differentiation of single bronchioalveolar stem cells during lung repair. Cell Discov. 2020;6(1). doi: 10.1038/s41421-019-0132-8.

3. Hagar M, Ahmed HA, Aljohani G, Alhaddad OA. Investigation of some antiviral $\mathrm{n}$ - heterocycles as Covid 19 drug: molecular docking and DFT calculations. Int J Mol Sci. 2020;21(11):3922. doi: 10.3390/ijms21113922, PMID 32486229.

4. Weniger H. Review of side effects and toxicity of chloroquine. Boll World Health. 1979;79:906.

5. Collins KP, Jackson KM, Gustafson DL. Hydroxychloroquine: A Physiologically-Based Pharmacokinetic Model in the Context of CancerRelated Autophagy Modulation. J Pharmacol Exp Ther. 2018;365(3):447-59. doi: 10.1124/jpet.117.245639, PMID 29438998.

6. Bernstein HN. Ocular safety of hydroxychloroquine. Ann Ophthalmol. 1991;23(8):292-6. PMID 1952638.

7. Easterbrook M. The ocular safety of hydroxychloroquine. Semin Arthritis Rheum. 1993;23(2);Suppl 1:62-7. doi: 10.1016/s0049-0172(10)80009-5, PMID 8278820.

8. Munster T, Gibbs JP, Shen D, Baethge BA, Botstein GR, Caldwell J, et al. Hydroxychloroquine concentration-response relationships in patients with rheumatoid arthritis. Arthritis Rheum. 2002;46(6):1460-9. doi: 10.1002/ art.10307, PMID 12115175.

9. Schrezenmeier E, Dörner T. Mechanisms of action of hydroxychloroquine and chloroquine: Implications for rheumatology. Nat Rev Rheumatol. 2020;16(3):155-66. doi: 10.1038/s41584-020-0372-x, PMID 32034323.

10. De Duve C. Lysosomes revisited. Eur J Biochem. 1983;137(3):391-7. doi: 10.1111/j.1432-1033.1983.tb07841.x, PMID 6319122.

11. Lu S, Sung T, Lin N, Abraham RT, Jessen BA. Lysosomal adaptation: How cells respond to lysosomotropic compounds. PLOS ONE. 2017;12(3):e0173771. doi: 10.1371/journal.pone.0173771, PMID 28301521.

12. Iredale J, Fieger H, Wainer IW. Determination of the stereoisomers of hydroxychloroquine and its major metabolites in plasma and urine following a single oral administration of racemic hydroxychloroquine. Seminars in Arthritis and Rheumatism. 1993;23(2):74-81. doi: 10.1016/S0049-0172(10)80011-3.

13. Browning DJ. Pharmacology of chloroquine and hydroxychloroquine, in hydroxychloroquine and chloroquine retinopathy. New York: Springer-Verlag; 2014. p. 35-63.

14. Chhonker YS, Sleightholm RL, Li J, Oupický D, Murry DJ. Simultaneous quantitation of hydroxychloroquine and its metabolites in mouse blood and tissues using LC-ESI- MS/MS: an application for pharmacokinetic studies. J Chromatogr B Analyt Technol Biomed Life Sci. 2018;1072:320-7. doi: 10.1016/j.jchromb.2017.11.026, PMID 29207305.

15. Frisch MJ, Trucks GW, Schlegel HB, et al. Wallingford, CT: Gaussian, Incorp; 2009.

16. Daina A, Michielin O, Zoete V. SwissADME: A free web tool to evaluate pharmacokinetics, drug-likeness and medicinal chemistry friendliness of small molecules. Sci Rep. 2017;7:42717. doi: 10.1038/srep42717. PMID 28256516.

17. Kumar SA, Bhskar BL. Preliminary investigation of drug impurities associated with the anti-influenza drug favipiravir - an in silico approach. Comput Theor Chem. 2021;1204. PMID 113375.

18. Noureddine O, Issaoui N, Al-Dossary O. DFT and molecular docking study of chloroquine derivatives as antiviral to coronavirus COVID-19. J King Saud Univ Sci. 2021;33(1):101248. doi: 10.1016/j.jksus.2020.101248.

19. Anil Kumar S, Bhaskar B. Spectroscopic and Computational approach for the Structure - Property relationship of Hydantoin drug impurity: 1-methyl5,5-diphenylimidazolidine-2, 4-dione. IOP Conf Ser.: Mater Sci Eng;577. doi: 10.1088/1757-899X/577/1/012180.

20. Parr RG, Szentpály Lv, Liu S. Electrophilicity Index. J Am Chem Soc. 1999;121(9):1922-4. doi: 10.1021/ja983494x.

21. Bendjeddou A, Abbaz T, Gouasmia A, Villemin D. Studies on chemical reactivity of $p$ - aminophenyl benzene-fused bis tetrathiafulvalenes through quantum chemical approaches. Am. J Appl Chem. 2016;4:104-10.

22. Kumar SA, Bhaskar BL. Computational and spectral studies of 3,3'-(propane1,3-diyl)bis(7,8-dimethoxy-1,3,4,5-tetrahydro-2H-benzo[d]azepin-2-one). 
Heliyon. 2019;5(9):e02420. doi: 10.1016/j.heliyon.2019.e02420, PMID 31687545.

23. Hansch C, Dunn III WJ. Linear relationships between lipophilic character and biological activity of drugs. J Pharm Sci. 1972;61(1):1-19. doi: 10.1002/ jps.2600610102, PMID 4550859.
24. Arnott JA, Planey SL. The influence of lipophilicity in drug discovery and design. Expert Opin Drug Discov. 2012;7(10):863-75. doi: 10.1517/17460441.2012.714363, PMID 22992175.

25. Bhal SK, Log P. Making sense of the value. Advanced Chemistry Development, Inc (ACD Labs). (Available from: http://www.acdlabs.com/ resources/knowledgebase/app_notes/-physchem/making_sense.php.

\section{PICTORIAL ABSTRACT}
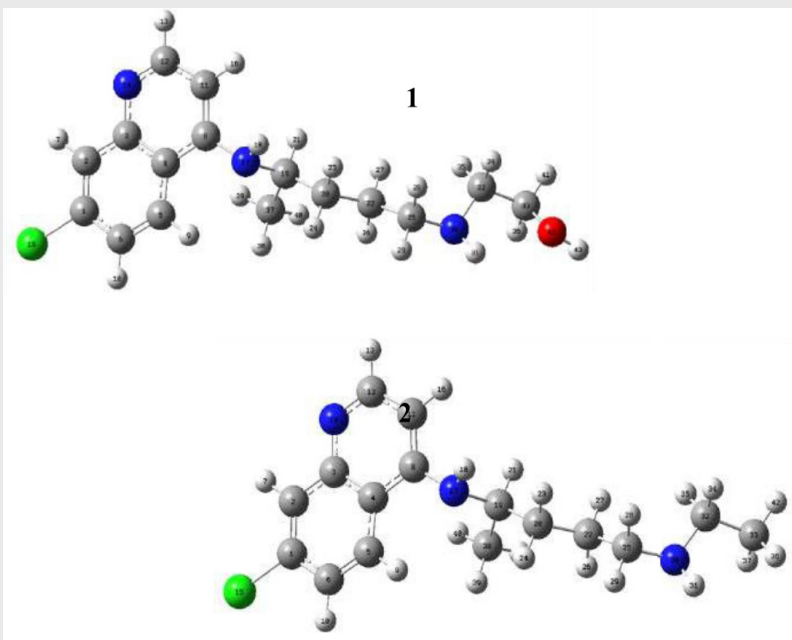

3

Cite this article: Islam MM, Singh PS, Rushd S. Hydroxychloroquine Metabolites - An Exploratory Computational Study. Indian J of Pharmaceutical Education and Research. 2022;56(1):215-23. 